\title{
Impacto en la salud por el inadecuado manejo de los residuos peligrosos
}

\author{
Mireya Tovar Artunduaga \\ Universidad Cooperativa de \\ Colombia. \\ mtovar5@gmail.com
}

\author{
Gloria M. Losada Salazar \\ Universidad Cooperativa de \\ Colombia. \\ glomelosa_584@hotmail.com
}

\author{
Tatiana F. García \\ Universidad Cooperativa de \\ Colombia. \\ Tatiana.garciar@campusucc.edu.co
}

(Tipo de Artículo: Revisión. Recibido el 27/04/2015. Aprobado el 06/08/2015)

\begin{abstract}
Resumen. Desde hace algunos años en Colombia se vienen implementando convenios y normas para el adecuado manejo y disposición final de los residuos o desechos peligrosos (RESPEL), que permitan reglamentar la conducción eficiente de residuos generados en gran cantidad por las empresas y población en general. De acuerdo a investigaciones realizadas por el Ministerio de Salud, se puede evidenciar que el inadecuado manejo de estos residuos traen como consecuencia efectos negativos en la salud que van desde simples laringitis hasta enfermedades más complejas como el cáncer; debido a que la gran mayoría de procesos productivos y de consumo son arrojados al ambiente tales como: mercurio, plomo, plaguicidas, entre otros. El cumplimiento estricto de las normas minimizaría el costo en materia de salud y medio ambiente.
\end{abstract}

Palabras Claves. Residuo o Desecho Peligroso, Contaminación, Salud.

\section{Health impact by improper management of hazardous waste}

\begin{abstract}
For some years in Colombia has been implementing agreements and standards for proper handling and disposal of waste or hazardous waste (RESPEL), enabling efficient driving regulate waste generated in large quantities by companies and the general population. According to research conducted by the Ministry of Health, one can show that the inadequate management of these wastes resulting bring negative health effects ranging from simple laryngitis to more complex diseases such as cancer; because the vast majority of production and consumption processes are thorwn into the atmosphere tale as mercury, lead, pesticides, among others. Strict compliance minimize the cost in health and environment.
\end{abstract}

Keywords. Waste or Hazardous Waste, Pollution, Health.

\section{INTRODUCCIÓN}

La mayor parte de las acciones que realiza el ser humano diariamente ya sea por causa del trabajo o de las diferentes actividades propias de su supervivencia, genera una serie de residuos de variada naturaleza; estos residuos se producen en diferentes estados (sólidos, líquidos y/o gaseosos). Algunos de estos residuos debido a su composición química, física y/o biológica provocan efectos adversos de diversa magnitud al hombre y al medio ambiente. Estos se conocen como residuos o desechos peligrosos.

En Colombia y en el mundo los desechos peligrosos contaminan el aire, el agua o el suelo, debido a la gestión inadecuada de las actividades productoras (agropecuarias, mineras, hospitalarias, manufactureras), labores domésticas (no clasificación de los residuos en la fuente) e Instituciones prestadoras del servicio de recolección que no abastecen el $100 \%$ de la demanda.

Dichas condiciones afectan considerablemente el medio ambiente y la salud de la población, especialmente los grupos vulnerables como: niños, mujeres gestantes, población adulta mayor y población en extrema pobreza.

Las afectaciones generadas por la contaminación de los recursos naturales, se convierten en los problemas de más alto costo para la economía del país, según los datos proporcionados en un estudio denominado "Diagnóstico y el estudio de costos de la degradación ambiental del Análisis Ambiental País - AAP" [1] debido al trabajo de recuperación de los señalados recursos, así como también, la recuperación de la salud de la población a afectada.

Es por eso que es necesario informar a la población en general del alto riesgo que se corre a causa de la contaminación, y una manera de hacerlo es a través de artículos informativos como este, en el que se notifican cifras puntuales de investigaciones hechas donde se demuestra el riesgo para la salud y el medio ambiente debido al inadecuado manejo de residuos peligrosos.

\section{MANEJO DE LOS RESIDUOS PELIGROSOS EN COLOMBIA}

Colombia es un país en vía de desarrollo con una importante actividad agrícola e industrial que consume grandes volúmenes de productos químicos de grado variable de toxicidad. Es así, como para el año 2010 se estima que en el país se produjeron un poco más de 24.000 toneladas y 25 millones de litros de plaguicidas, y se importaron alrededor de 53.000 toneladas de los mismos, además de la producción de medicamentos, cosméticos, hidrocarburos, entre otros [2].

Adicionalmente se calcula que el país produce anualmente un promedio de 400.000 toneladas al año de residuos peligrosos procedentes en su mayoría de la fabricación de sustancias y productos químicos derivados del petróleo y del carbón, de caucho y plástico, de la industria metalúrgica, de la industria de minerales no 
metálicos, hospitales, la industria energética, farmacéutica y textil [3].

De acuerdo a los datos mencionados anteriormente, se debe considerar el impacto que causa en la salud de la población la mala manipulación de los productos químicos utilizados por la industria y su disposición final, ya que en el estudio de carga de enfermedad elaborado por la Organización Mundial de la Salud (OMS) en el año 2004, se indica que de las 102 enfermedades principales, 85 de ellas eran en parte causadas por la exposición a riesgos ambientales y que además los factores ambientales contribuían con alrededor de una cuarta parte de los años de vida perdidos ajustados en función de la discapacidad, así como a una cuarta parte de los fallecimientos relacionados [4].

Además la Organización Panamericana de la Salud (OPS), a su vez, estima que el $24 \%$ de la carga mundial de morbilidad y $23 \%$ de todas las defunciones pueden atribuirse a factores relacionados con el ambiente. Para los países en desarrollo el porcentaje de mortalidad atribuible a causas ambientales es de $25 \%$, y en los desarrollados de $17 \%$, la cual puede estar relacionada con la inequidad en el acceso a servicios públicos, la falta de ingresos y el crecimiento acelerado del sector industrial, factores que incrementan los niveles de vulnerabilidad y exposición de la población; esto genera un impacto negativo en los niveles de carga de enfermedad [5].

Adicionalmente, se reportan para Colombia 46.000 defunciones al año atribuibles a condiciones ambientales; los factores ambientales que más contribuyen a muertes prematuras son la contaminación del aire en exteriores e interiores y las condiciones del agua, saneamiento e higiene, esto debido a que el vertimiento de aguas residuales de los diferentes procesos productivos, incluyendo las domesticas, de las cuales solo el $10 \%$ de ellas son tratadas, generando un gran impacto en el medio ambiente y la salud humana [6].

Lo anteriormente mencionado, deja ver la problemática a nivel socioeconómico que debe enfrentar el país en materia de costos, pues de acuerdo al estudio realizado por Larsen [7] para el Ministerio de Ambiente, Vivienda y Desarrollo Territorial (MAVDT), hoy Ministerio de Ambiente y Desarrollo Sostenible, en donde se estimaron los costos sociales y económicos del deterioro ambiental en Colombia, se observa que "los efectos negativos en la salud relacionados con la degradación ambiental son superiores a los de otros países con niveles de ingreso similar, alcanzando un costo en mortalidad y morbilidad del $2.8 \%$ del Producto Interno Bruto (PIB)", aproximadamente 5.2 billones de pesos al año.

\subsection{Efectos en la salud por la contaminación del aíre}

Cabe mencionar que los factores contaminantes nocivos para la salud y que deterioran de manera considerable el aire se pueden presentar de diferentes formas en el ambiente, como los gases, el humo, el polvo y las partículas, los cuales son generados por fuentes externas o internas donde se desarrollan e interactúan los individuos. El estudio "Cost of Environmental Damage: $A$ Socio-Economic and Environmental Health Risk Assessment" elaborado por Bjorn Larsen en el año 2004 [7], presenta evidencias sobre cómo el contaminante más fuertemente asociado a las muertes prematuras, bronquitis y afecciones respiratorias es el material particulado, en especial las partículas de menos de 2.5 micras de diámetro (PM2.5).

Contando con las afirmaciones de Bjorn Larsen [7]. en su estudio para nuestro país, es de gran importancia vigilar la emisión de gases y partículas al medio ambiente, ya que estos factores nos pueden llevar a continuar con daños a la salud como los que indica Sánchez Triana en su estudio "Prioridades ambientales para la reducción de la pobreza en Colombia, un análisis ambiental del país para el Banco Mundial" [1], en donde se calcula que aproximadamente 6.000 muertes y 7.400 nuevos casos de bronquitis crónica anuales son atribuibles a la calidad del aire en exteriores. Además Sánchez Triana [1], nos muestra que más del $30 \%$ de los casos se presentan en Bogotá y más del $20 \%$ en ciudades con menos de un millón de habitantes. Por otro lado, se estima que cerca de 1.000 muertes al año son atribuibles a la calidad del aire en interiores por el uso de combustibles sólidos [1].

La exposición prolongada a un contaminante puede reducir en varios años la esperanza de vida de un ser humano, esto se ve reflejado en el incremento de los ingresos hospitalarios y en el registro de más casos de enfermedades cardiovasculares y pulmonares en el mundo, le emisión de dióxido de carbono y otros contaminantes son causantes de que aproximadamente más de siete millones de personas mueran cada año.

Adicionalmente se considera que la contaminación del aire afecta más a países industrializados, pero no es así porque un $80 \%$ de las muertes en el mundo, por esta contaminación, se producen en países donde existe la pobreza, en donde las comunidades más vulnerables y pobres utilizan las energías que más contaminan, como el carbón, el estiércol y la leña, para cocinar o calentarse dentro de sus hogares [1].

Adicionalmente, Aristizabal [8] describe que dentro de los efectos crónicos nocivos, producidos por los contaminantes en el aparato respiratorio, se pueden encontrar la alteración estructural e inhibición del sistema mucociliar, hiperplasia celular epitelial e inflamación de la mucosa respiratoria, y como efectos agudos pueden considerarse la faringitis, laringitis, traqueítis y la neumonía, donde la población más susceptible son los niños menores de 5 años.

Conforme a lo anterior, Shah y Kojima han estimado que toda acción orientada a reducir en un $50 \%$ la carga de sulfatos y partículas suspendidas en el aire de las áreas urbanas, podrían contribuir a reducir la tasa total de mortalidad en un 4,7\%, y así lograr un aumento de la 
expectativa de vida hasta en 9.6 meses [9]. Teniendo en cuenta que los datos relacionados en el presente artículo hacen referencia a investigaciones realizadas hace más de 10 años, surge la necesidad de hacer nuevos estudios investigativos que permitan verificar las acciones orientadas a reducir las partículas contaminante en el aire, pues a simple vista podemos observar que las enfermedades van en aumento, por lo que se podría presumir que las acciones de entes gubernamentales no están siendo efectivas a la hora de hacer cumplir la normatividad legalmente constituida.

\subsection{Efectos en la salud a causa de sustancias Químicas}

Otro de los factores contaminantes del medio ambiente y que afecta la salud humana son las sustancias químicas, estas sustancias son utilizadas en todas las actividades productivas del hombre, en diferentes cantidades; pero en Colombia su incidencia en la salud se basa en las intoxicaciones y muertes que se producen anualmente, derivadas de la inadecuada manipulación de los plaguicidas y su uso por personas sin entrenamiento adecuado, además de la contaminación que originan al medio ambiente, ha hecho que estos productos sean considerados como un problema grave de alto impacto en salud pública. Según la Organización Mundial de la Salud (OMS) en el año 2006, la exposición a sustancias químicas da cuenta de más del $25 \%$ de las intoxicaciones y el $5 \%$ de los casos de enfermedades como cáncer, desórdenes neuropsiquiátricos y enfermedades vasculares a nivel mundial [10].

Desde el año 2003, Colombia cuenta con un sistema de vigilancia epidemiológica para las intoxicaciones por sustancias químicas, el cual fue reglamentado en el año 2006, mediante la creación del Sistema Nacional de Vigilancia en Salud pública-Sivigila, definiendo claramente la vigilancia rutinaria de intoxicaciones agudas por plaguicidas [11]. Durante el período del 2010 al 2012 se notificaron al Sivigila 23.844, 27.126 y 27.252 casos de intoxicaciones por sustancias químicas (plaguicidas, medicamentos, metanol, metales pesados, solventes, gases y otras sustancias químicas), respectivamente. Para el 2013, el mayor porcentaje de intoxicaciones por sustancias químicas correspondieron a intoxicaciones por medicamentos $(32,13 \%)$, seguidos de intoxicaciones por plaguicidas $(29,17 \%)$ e intoxicaciones por sustancias psicoactivas (16,94\%); concentrando el mayor número de eventos en las entidades territoriales de Bogotá, Antioquia, Valle, Nariño y Huila [12]. Sin embargo, en la actualidad no existe la forma de poder cuantificar los datos reales de los niveles de exposición de las personas en Colombia que manejan inadecuadamente los plaguicidas, ya que los casos que se reportan en el Sivigila hacen parte de casos de intoxicación extrema sin considerarse las personas que tienen contactos permanentes con algunas sustancias (plaguicidas, herbicidas) que a largo plazo pueden generar efectos negativos en la salud.
Considerando los efectos en la salud que causan los desechos peligrosos, para el año 2012 en Colombia, la generación de residuos o desechos peligrosos fue de 250.698,3 toneladas, de acuerdo al reporte realizado al Instituto de Hidrología, Meteorología y Estudios Ambientales de Colombia (IDEAM), cabe mencionar que el Registro de Generadores de Residuos o Desechos Peligrosos [13] es diligenciado vía Web directamente por los establecimientos generadores de este tipo de residuos a nivel nacional; las autoridades ambientales son responsables de la revisión de la información consignada por los generadores y de realizar la transmisión de dicha información al IDEAM, dando cumplimiento a la Resolución 1362 del 2 de Agosto de 2007 [14] expedida por el Ministerio de Ambiente, Vivienda y Desarrollo Territorial, hoy Ministerio de Ambiente y Desarrollo Sostenible, con base en lo estipulado en el Artículo 27 del Decreto 4741 del 30 de Diciembre de 2005 [15] Teniendo en cuenta la actividad productiva, la extracción de petróleo crudo fue la que aportó la mayor cantidad de residuos peligrosos, con el $46,8 \%$ del total de los residuos o desechos peligrosos generados en dicho año; en segundo lugar las industrias básicas del hierro y del acero, con el $7,2 \%$ del total generado en 2012 y en tercer lugar las actividades de hospitales y clínicas con internación, con el $7,0 \%$ de la generación nacional en el mismo período [13].

De acuerdo al reporte de los residuos peligrosos ya expuestos, llama la atención el hecho que más del 35\% de los residuos peligrosos que fueron llevados a disposición final en 2012 aparezcan reportados como dispuestos en sitios diferentes a una celda de seguridad o a un relleno de seguridad, ya que son estos dos tipos de instalaciones los únicos autorizados en el país para realizar confinamiento de residuos o desechos peligrosos [13]. Lo anterior puede deberse a que algunos generadores no están haciendo un reporte adecuado en el apartado de disposición de residuos del Registro de Generadores de Residuos o Desechos Peligrosos, y están declarando el manejo de algunas corrientes de residuos por disposición final, siendo que las están manejando por medio de una alternativa de manejo diferente. Esta situación debe despertar especial interés por parte de las Autoridades Ambientales donde los generadores están reportando este tipo de disposición final, con el fin de garantizar que los residuos peligrosos generados en su jurisdicción, están recibiendo una disposición final adecuada y con ello lograr preservar la salud humana y el ambiente. Adicionalmente cabe mencionar que para el 31 de diciembre de 2012, se encuentra un total de $23.304,2$ toneladas de residuos o desechos peligrosos que no habían sido gestionadas de ninguna manera y se hallaban almacenadas en las instalaciones del generador o en instalaciones de un tercero que presta el servicio de almacenamiento [13].

Las autoridades ambientales deben de contar con los recursos físicos, humanos, tecnológicos y financieros 
necesarios para ejercer control y vigilancia sobre los entes generadores de residuos peligrosos y aplicar la Ley de forma equitativa tanto para el gran generador como para los no obligados a reportar, esto con base a que cada habitante de nuestro país debe considerarse como generador de residuos peligrosos puesto que estos adquieren bienes de consumo con un grado potencial de peligrosidad, de acuerdo a los principios activos de los cuales están compuestos, puesto que dicha composición está dada básicamente por sustancias químicas o elementos que se deben manejar adecuadamente cuando culmina su vida útil, tal es el caso de los elementos de limpieza y aseo, aparatos electrónicos y electrodomésticos, plaguicidas, pinturas y barnices en general, medicinas y medicamentos, productos para los automóviles. De estos elementos de consumo se derivan bombillos y lámparas fluorescentes, pilas y baterías, cilindros de gas, residuos eléctricos y electrónicos, tóner y cartuchos vencidos, medicamentos vencidos, envases contaminados, trapos contaminados, residuos biosanitarios, entre otros, los cuales no se deben mezclar con los residuos orgánicos de cada vivienda ya que estos deben de tener un tratamiento especial debido a la contaminación que pueden causar.

\section{CONCLUSIONES}

En vista de lo anterior, se concluye que a nivel Nacional, los diferentes Municipios están lejos de cumplirle a los Colombianos con tener un País más sano y amigable con el medio ambiente, sin calentamiento global, como está pasando en la actualidad, ya que las ciudades con altos estándares industriales no asumen con seriedad los compromisos, para rebajar la producción de sustancias peligrosas, que ellos mismos generan.

Así mismo, se debe reconocer que el tema de los Residuos peligrosos es de especial importancia para Colombia, por los efectos y riesgos potenciales para la salud humana y el medio ambiente, en ese sentido Colombia también ha sido responsable ya que las diferentes Corporación Autónoma Regionales Departamentales, vienen apoyando a los diferentes municipios del país, en la Ejecución del "Plan Departamental para la Gestión Integral de Residuos o Desechos Peligrosos RESPEL.

Por otro lado en nuestro país además de lo anterior, se tendrá que entrenar adecuadamente a un sector de la población muy vulnerable, los campesinos, quienes debido a su actividad económica usan demasiados agroquímicos, y en muchos casos desconocen el daño que generan dichos componentes a su organismo y por el aire se arremete con fumigaciones, produciendo deforestación, contaminación del medio ambiente, destrucción de la capa de ozono, ya que estos gases también van a la atmosfera y producen este efecto. Y si lo anterior no fuera suficiente la explotación del petróleo, con la sísmica usada actualmente por las diferentes compañías petroleras, también es desastrosa degrada la tierra en todas sus formas. Razón por la cual, Colombia debe cambiar esas condiciones que afrontan nuestros campos, metiendo en cintura a las compañías de explotación minera en general para que cumplan con los estándares de calidad de producción y cumplan con los parámetros que exige la Ley.

Pero en general, en Colombia se evidencia la carencia de sistemas nacionales de información y seguimiento, esta falta restringe la posibilidad de planificar y de contar con un elemento valioso para la correcta toma de decisiones, la adecuada gestión, la formalización de planes y programas, la jerarquización de actividades, la asignación de recursos y la realización de labores de monitoreo, vigilancia y control. Adicional a esto la legislación es inadecuada en algunos casos, debido a que no existe coherencia entre las disposiciones jurídicas referidas a los residuos sólidos peligrosos y los riesgos que representan para la salud pública y el ambiente. La legislación es incompleta y ambigua respecto al ámbito de competencia de las instancias administrativas involucradas y es incompatible con las situaciones económicas, sociales y culturales imperantes, además hay abuso en la expedición y empleo de disposiciones complementarias y administrativas. La mayoría de las ciudades no contempla los múltiples compromisos internacionales asumidos por el Gobierno, y si los contempla, no se aplican a la realidad.

El inadecuado manejo de los residuos o desechos peligrosos ha sido la principal causa en Colombia de los grandes problemas de contaminación y de salud pública, la gran mayoría concluyen que se debe a la falta en el cumplimiento de los protocolos, ya que las entidades públicas y privadas, no cuenta con un procedimiento adecuado para la manipulación y disposición final de los residuos sólidos y líquidos siendo estos vertidos al sistema de alcantarillado, y las partículas y gases son suspendidos en el aire sin ninguna medida de control, incidiendo esto negativamente en el medio ambiente y también en el campo Social, Económico y Político, dándose esto último, a pesar de existir políticas claras, dentro de la legislación Colombiana, pero que no han sido implementadas en forma eficiente.

Culturizar y entrenar a la población en general que manipula o están expuestas a residuos o desechos peligrosos por ocasión de su trabajo o por utilización de elementos de consumo, para que ellos no sufran las consecuencias de la falta de conocimiento de los riesgos a los que están expuestos, y las enfermedades a las que se pueden enfrentar más adelante, ya sea por exposición al riesgo o por la contaminación de los recursos naturales del hábitat donde viven. 


\section{REFERENCIAS}

[1] E. Sánchez Triana, K. Ahmed, Y. Awe, "Prioridades ambientales para la reducción de la pobreza en Colombia. Un análisis ambiental del país para Colombia", Banco Internacional de Reconstrucción y Fomento/ Banco Mundial, Washington D.C, 2007.

[2] S.P. Hurtado Serrano, "Estadísticas de comercialización de plaguicidas químicos de uso agrícola 2010", Instituto Colombiano Agropecuario ICA, Bogotá D.C., Boletín Técnico No.00.02.78.11, Diciembre 2011.

[3] Política Ambiental para la Gestión Integral de Residuos o Desechos Peligrosos, $1^{\text {ra }}$ ed., Ministerio de Ambiente Vivienda y Desarrollo Territorial MAVDT, Bogotá D.C., 2005, pp 8-122.

[4] Organización de las Naciones Unidas, Programa para el medio Ambiente PNUMA (2015). [En línea]. Disponible:http://www.dicc.hegoa.ehu.es/listar/mostrar/171

[5] Documento Conpes, 3550, 2008,

[6] J. Estevez, "Avances Sectoriales en la implementación de la política integral de salud ambiental", Congr. Int. de Salud Pública. Medellín, 2011, pp 1-28.

[7] B. Larsen, "Costos de los daños al medio ambiente: Una Evaluación de los riesgos de la Salud Socio-económico y ambiental", Ministerio de ambiente, Vivienda y Desarrollo Territorial, Bogotá D.C., 2004.

[8] G. Aristizabal, J. Suescun, R. Patiño, "Contaminación del aire y enfermedad respiratoria en población infantil de Puente Aranda", Rev. Salud Pública, vol.15, no 4, pp 503516, Agosto, 2013.

[9] J.J. Shah, T. Nagpal y C. Brandon, "Urban Air Quality Management Strategy in Asia". Banco Internacional de Reconstrucción y fomento/Banco mundial, Washington D.C, 1997.

[10] A. Prüss-Ustün, and C. Corvalan, "Preventing disease through healthy environments. Towards an estimate of the environmental burden of disease", World Health Organization, Washington D.C., 2006.

[11] Decreto, 3518, 2006.

[12] H. Quijada Bonilla, "Informe de intoxicaciones por sustancias químicas", Ministerio de Salud, Instituto Nacional de Salud, Bogotá D.C., 2013.

[13] O. Franco Torrres, "Informe Nacional Generación y Manejo de Residuos o Desechos Peligrosos en Colombia 2012" Instituto de Hidrología, Meteorología y Estudios Ambientales de Colombia, Bogotá D.C., Marzo de 2014.

[14] Resolución, 1362, 2007.

[15] Decreto, 4741, 2005. 